\title{
Viewpoint \\ When will tumor gene expression profiling be incorporated into clinical breast cancer decision making?
}

Chad J Creighton ${ }^{1}$ and James M Rae ${ }^{2}$

\author{
${ }^{1}$ Bioinformatics Program, University of Michigan Medical Center, Ann Arbor, Michigan, USA \\ 2Division of Hematology Oncology, Department of Internal Medicine, University of Michigan Medical Center, Ann Arbor, Michigan, USA
}

Corresponding author: James M Rae, jimmyrae@umich.edu

Published: 11 July 2006

This article is online at http://breast-cancer-research.com/content/8/4/302

(c) 2006 BioMed Central Ltd

Breast Cancer Research 2006, 8:302 (doi:10.1186/bcr1519)

\begin{abstract}
DNA microarray based gene expression analysis of primary breast cancers is not in general clinical use in spite of the widespread enthusiasm for the approach. However, several studies have identified gene expression patterns, or "signatures" that are predictive of disease outcome and assays based on these findings have become commercially available. Additional studies are required to further define gene signatures that can be used to help tailor the therapy best suited to for an individual patient. Recent gene expression profiling studies using cell line models to identify downstream transcriptional targets of oncogenic signaling pathways may help achieve this goal.
\end{abstract}

Gene expression profiling of cancers has great potential as a valuable tool in the identification of new clinical biomarkers and of the molecular pathways that underlie the disease. Among other factors, expression profiling may uncover new molecular subtypes of breast cancer that transcend current histologic definitions. In a series of seminal studies conducted by Sorlie and coworkers [1], hierarchical clustering of breast tumor profiles from multiple independent datasets revealed a basal epithelial-like group, an ERBB2/HER2overexpressing group, two distinct luminal-like groups, and a normal breast-like group. However, current histologic definitions of breast cancer largely reflect the tumor subtypes uncovered by Sorlie and colleagues; for instance, the basal tumors reflect the estrogen receptor (ER)-negative subtype, whereas the luminal tumors reflect the ER-positive subtype. It would be useful to know whether additional relevant subtypes of breast cancer may be uncovered, either by profiling larger cohorts of tumors or by using alternative data analysis approaches, with the ultimate goal of individualizing therapy based on the molecular profile of the patient's tumor. There is already established precedence for clinical use of molecular markers to help make decisions on the course of treatment in breast cancer, because expression of ER and progesterone receptor are used to assess the potential response to hormonal therapy, and expression of ERBB2/HER2 is used to assess potential response to herceptin.

A number of studies have identified gene expression patterns in breast cancer with the ability to predict disease outcome. The first of these studies is that by van 't Veer and coworkers [2], in which a classifier of 70 genes was defined that outperformed all clinical variables in predicting the likelihood of distant metastases within 5 years. Van't Veer and colleagues have formed a cancer diagnostics company [3], which provides a service called MammaPrint to assess recurrence risk in breast cancer patients using cDNA microarrays to profile the 70 -gene set. The service is intended to assist in ensuring that those patients who are at high risk for metastases receive additional therapy designed to reduce the chance of disease recurrence. In addition, the MammaPrint service is intended to identify and spare low risk patients from receiving unnecessary chemotherapy, avoiding unnecessary costs and potentially serious side effects. The clinical utility of Mammaprint is being tested in a prospective phase III study (MINDACT); however, the utility and effectiveness of the test will not be known for many years.

Although much enthusiasm initially surrounded the profiling studies from van 't Veer and others, a substantial amount of skepticism has since emerged in the breast cancer community regarding whether gene signatures predictive of breast cancer outcome will eventually be incorporated into clinical breast cancer decision making. One set of issues surrounds statistical concerns that prognostic gene signatures such as the van't Veer 70-gene set were developed using training datasets representing a single cohort of a relatively small number of patients, and so therefore they may not be universally applicable to breast cancer patients. Additionally, some of the prognostic gene signatures derived for various cancers were 'validated' on

$\mathrm{ER}=$ estrogen receptor; $\mathrm{RT}-\mathrm{PCR}=$ reverse transcriptase polymerase chain reaction. 
subsets of the same patient cohort used to generate the given signature. Furthermore, gene expression profiling studies of breast cancer subsequent to that of van 't Veer and coworkers have derived top sets of prognostic markers that differ somewhat from the 70-gene set; in our opinion, however, this last issue does not point to any inconsistency in independently obtained results but rather to the fact that the entire population of genes correlated with patient survival may be quite large. At the same time, one could argue that the 70-gene set, for example, may not necessarily represent the 'best' or optimal set of predictors, and many have advocated using training datasets of thousands, rather than tens, of patient profiles for developing more robust prognostic signatures. Importantly, many signatures such as the van 't Veer signature have been shown to be prognostic in multiple microarray datasets from independent studies, although the predictive power, while statistically significant, has not been as impressive in terms of a hazard ratio in subsequent datasets.

Aside from statistics related issues, another issue with applying results of gene expression profiling studies to the clinic pertains to the distinction between general prognosis and therapeutic prediction. The gene signatures correlated with survival arising from previous profiling studies appear to be prognostic - that is, they indicate whether or not a patient will have a good outcome - but they do not necessarily predict how a patient will respond to a given course of adjuvant treatment. There is a need to develop gene signatures that may help in planning regimens of adjuvant therapy that are best suited to each particular patient.

Where the MammaPrint assay was designed for assessing prognosis in breast cancers, the commercially available Oncotype DX gene assay [4] was originally designed and extensively validated for predicting response to tamoxifen therapy in ER-positive breast cancer patients. The assay measures the expression levels of 21 genes by RT-PCR of RNA from paraffin-embedded tissues. As described in the study by Paik and coworkers [5], the 21 genes, 16 of which are cancer related and the other five of which are reference genes, were prospectively selected using the biomedical literature, gene expression array datasets, and RT-PCR assays of multiple independent clinical cohorts. The Oncotype DX assay has a distinct advantage over the MammaPrint assay in being able to use paraffin-embedded tissues, thereby having no extra requirements for tumor RNA sample procurement. Recently, Medicare agreed to cover the costs of Oncotype DX beginning late February of 2006. Oncotype DX has recently been shown not only to predict response to tamoxifen but also to indicate prognosis in untreated patients.

In addition to using gene expression profiling to assess the potential overall response to therapy, tumor profiling may predict response to neoadjuvant therapy, as demonstrated in a study by Chang and coworkers [6]. In this study, core biopsy samples were taken from primary breast tumours in 24 patients before treatment with four cycles of docetaxel over 3 weeks. The samples were profiled and the expression pattern of 92 genes found to distinguish responsive verses nonresponsive tumors. We believe that this approach could be expanded to include additional therapies with the ultimate goal of classifying cancers into 'therapeutic responsive' groups instead of the current 'pathological' groups. Identifying patients who are likely to respond to a particular therapy before treatment will help to minimize some of the unavoidable trial and errors currently associated with clinical oncology. In the case of molecular targeted therapies such as tamoxifen or herceptin, it may be possible to determine whether the therapy is having any impact on the pathway of interest, as 'oncogenic mRNA signatures' (described below) of signaling pathways such as ER or HER2 become available.

In order to develop more effective treatments for breast cancer, we need a better understanding of the molecular pathways that underlie the development and progression of the disease. RNA expression profiling studies have uncovered hundreds of genes with correlations with clinical parameters such as ER status or patient outcome. However, to make sense of the bigger picture of breast cancer at a molecular level, we need to be able to associate the genes with the various pathways they represent. Although molecular biology has made great strides in the past 30 years in uncovering, at the signal transduction level, the oncogenic pathways on which different cancers rely, until recently we have had an incomplete understanding of what many of these pathways 'look' like at level of gene transcription. Numerous studies are now being published in which a given signaling pathway of interest is manipulated in cell culture or mouse models, in order to identify the downstream transcriptional targets of the pathway by expression profiling. With the data from these experiments being made publicly available, further studies could integrate these pathway mRNA signature data with breast tumor profile data in meaningful ways.

A recent study by Bild and coworkers [7] highlights how gene signatures of oncogenic pathways can be used to identify patterns of pathway deregulation in breast cancer cells, with implications for guiding targeted therapies. Using human primary mammary epithelial cell cultures and recombinant adenoviruses, those investigators activated the oncogenic pathways of Myc, Ras, E2F3, Src, and $\beta$-catenin. Using gene expression profiles of these cell culture experiments, human breast tumor profiles could be separated, on the basis of which pathways appeared to be activated, into categories relevant to clinical outcome. Furthermore, in a panel of breast cancer cell lines, the sensitivity of a given cell line to Ras pathway inhibitors could be predicted on the basis of whether the profile of the cell line shared similarities to the Ras oncogenic signature. This study further indicates that we may be approaching a time when, given an expression profile from a patient's tumor, we could make an accurate 
assessment in the clinic of which pathways would be best to target in that patient.

In conclusion, we believe that gene expression profiling will have increasing impact in the clinic over the next several years, in terms of our being better able to assess the risk of recurrence in breast cancer patients, to predict patient response to neoadjuvant therapy, to assess which oncogenic pathways are deregulated in a given tumor as a guide for targeted adjuvant therapy, and to develop better targeted therapies as our understanding of the molecular biology of breast cancers improves.

\section{Competing interests}

The authors declare that they have no competing interests.

\section{References}

1. Sorlie T, Perou CM, Tibshirani R, Aas T, Geisler S, Johnsen $H$, Hastie T, Eisen MB, van de Rijn M, Jeffrey SS, et al.: Gene expression patterns of breast carcinomas distinguish tumor subclasses with clinical implications. Proc Natl Acad Sci USA 2001, 98:10869-10874.

2. van 't Veer LJ, Dai H, van de Vijver MJ, He YD, Hart AA, Mao M, Peterse HL, van der Kooy K, Marton MJ, Witteveen AT, et al: Gene expression profiling predicts clinical outcome of breast cancer. Nature 2002, 415:530-536.

3. Agendia homepage [www.agendia.com]

4. Genomic health homepage [www.genomichealth.com]

5. Paik S, Shak S, Tang G, Kim C, Baker J, Cronin M, Baehner FL, Walker MG, Watson D, Park T, et al:: A multigene assay to predict recurrence of tamoxifen-treated, node-negative breast cancer. N Engl J Med 2004, 351:2817-2826.

6. Chang JC, Wooten EC, Tsimelzon A, Hilsenbeck SG, Gutierrez MC, Elledge R, Mohsin S, Osborne CK, Chamness GC, Allred $D C$, et al.: Gene expression profiling for the prediction of therapeutic response to docetaxel in patients with breast cancer. Lancet 2003, 362:362-369.

7. Bild AH, Yao G, Chang JT, Wang Q, Potti A, Chasse D, Joshi MB, Harpole D, Lancaster JM, Berchuck A, et al.: Oncogenic pathway signatures in human cancers as a guide to targeted therapies. Nature 2006, 439:353-357. 